Periódico do Núcleo de Estudos e Pesquisas sobre Gênero e Direito Centro de Ciências Jurídicas - Universidade Federal da Paraíba V. 7 - $\mathrm{N}^{\mathrm{o}} 04$ - Ano 2018 - Spanish Edition ISSN | 2179-7137 | http://periodicos.ufpb.br/ojs2/index.php/ged/index

\title{
DIFERENCIAS DE GÉNERO EN ESTILOS DE APRENDIZAJE Y RENDIMIENTO ACADÉMICO EN ESTUDIANTES DE ADMINISTRACIÓN
}

Resumen: El objetivo de esta investigación consiste en realizar una comparativa de las diferencias según el género en los estilos de aprendizaje en estudiantes de la Licenciatura en Administración, para ello se realizó un estudio de tipo descriptivo. La muestra estaba compuesta por 97 estudiantes de nuevo ingreso de los cuales 56 son del género femenino y 41 del género masculino, pertenecientes a las cohortes generacionales 2010, 2011 y 2012. A los cuales se les aplicó el inventario de Estilos de Aprendizaje y Orientación Motivacional (EDAOM). Los resultados obtenidos marcan la
María Arely López Garrido ${ }^{1}$ Erika Yunuen Morales Mateos ${ }^{2}$ Laura López Díaz ${ }^{3}$ Josefina De la Cruz Izquierdo ${ }^{4}$ pauta para determinar la existencia de diferencias entre los estilos de aprendizaje y la probable relación con el género. Al mismo tiempo, la investigación busca determinar si el género también tiene relación con el rendimiento académico que cada uno desarrolla y si se logran diferencias entre ellos.

Palabras Clave: Género, Estilos de aprendizaje, estudiantes

\begin{abstract}
The objective of this research is to make a comparison of the differences according to gender in the learning styles
\end{abstract}

\footnotetext{
${ }^{1}$ Dra. en Sistemas Computacionales. Profesora Investigadora de la División Académica de Ciencias Básicas. E-mail: arely.lopez@ujat.mx

${ }^{2}$ Dra. en Sistemas Computacionales. Profesora- Investigadora de la División Académica de Informática y Sistema E-mail: erika.yunuen@ hotmail.com

${ }^{3}$ Dra. en Educación. Profesora- Investigadora de la División Académica de Informática y Sistema E-mail: laliujat@hotmail.com

${ }^{4}$ Dra. en Educación. Profesora- Investigadora de la División Académica de Ciencias Sociales y Humanidades E-mail: Josefina.DelaCruz@hotmail.com
} 
of students of the Bachelor of Administration, for this a descriptive study was made, the sample was composed of 97 new students of which 56 are of the female gender and 41 of the male gender, belonging to the generational cohorts 2010, 2011 and 2012. To which the inventory of Learning Styles and Motivational Orientation (EDAOM) was applied. The results obtained set the pattern to determine the existence of differences between learning styles and the probable relationship with gender. At the same time, the research seeks to determine if gender is also related to the academic performance that each one develops and if differences are achieved between them.

KeyWords: Gender, Learning styles, students

\section{Introducción}

De acuerdo con la Real Academia Española el género es el grupo al que pertenecen los seres humanos de cada sexo, entendido este desde un punto de vista sociocultural en lugar de exclusivamente biológico (Real Academia
Española, s.f.).

Hay pocos estudios de la incidencia del género en el rendimiento académico, por lo cual no se conoce si hay diferencias en cuanto a las estrategias de aprendizaje empleadas (Meyer, 2015 citado en Cano, 2000).

Actualmente el rendimiento académico es un indicador de la calidad educativa muy importante para las universidades. Esta importancia se refleja en la influencia de manera indirecta en otros indicadores como son la eficiencia terminal y la reprobación. El rendimiento académico se puede definir como el cumplimiento de las metas, logros $\mathrm{u}$ objetivos de una asignatura que se está cursando (Vélez y Roa, 2005).

Las estrategias de aprendizaje son las actividades que el estudiante lleva a cabo para facilitar y mejorar la realización de las consignas determinadas por la asignatura, cualquiera que sea ésta, o por el contenido del aprendizaje. Los resultados del alto o bajo rendimiento se pueden concretar a través del uso apropiado de estrategias de aprendizaje (Beltran, 2003); y se pueden alcanzar mejores resultados del aprendizaje si se 
incorporan procedimientos de evaluación y análisis que permitan identificar riesgos y determinar las estrategias apropiadas que se deben de fomentar en los estudiantes acorde a sus estilos y características (Castañeda, 2004).

Un estudio realizado por Costa y Tabernero (2015), se propuso identificar y comprobar si existían diferencias de género en el rendimiento académico de estudiantes nivel secundaria. En este estudio se logró la participación de un total de 406 estudiantes; de dichos estudiantes, 190 pertenecían al género femenino y 215 al género masculino. Encontraron la existencia de ciertas diferencias que favorecían al género femenino, pero esta facilidad se presentaba en las asignaturas de Lengua Española., Literatura e Idioma Extranjero. Con referencia al autoconcepto positivo y en las facetas académica, social, emocional, familiar $\mathrm{y}$ física, no encontraron diferencias referentes al género.

El estudio realizado por Dona et al. (2010) se enfocaron en la realización de un estudio que permitiera la identificación de las estrategias de aprendizaje utilizadas por los estudiantes universitarios, clasificándolos de manera diferente acorde al género y la asociación entre las estrategias empleadas y el rendimiento académico. En este estudio se decidieron por aplicar el cuestionario utilizado por las Escalas de Estrategias de Aprendizaje ACRA. Para tal situación determinaron tomar una muestra, la cual estaba compuesta por 364 estudiantes de ambos sexos que pertenecían a la Universidad Nacional de la Plata. Implementaron estrategias enfocadas a las dimensiones de apoyo al aprendizaje y hábitos de estudio. Los resultados de dicha investigación demostraron que los estudiantes que contaban con mejores promedios implementaban una mayor cantidad de estrategias durante su trayecto educativo que aquéllos de menor promedio. Al igual que se destacó la observación de aplicación de diferentes estrategias de aprendizaje para los hombres, como para las mujeres.

El estudio realizado por Acevedo et al. (2015), se enfocó en el análisis de los estilos de aprendizaje en estudiantes pertenecientes a la Facultad de Ingeniería de la Universidad de Cartagena. Esta muestra estaba conformada por 144 
estudiantes, de los cuales son 72 hombres y 72 mujeres con edad promedio de 22 años. A dicha muestra se le pidió resolvieran el cuestionario Honey-Alonso que logra la identificación de los estilos de aprendizaje, clasificados como activo, reflexivo, teórico y pragmático. Para dicho análisis se determinó uno de varianza bifactorial y uno de correlación, pero en sus resultados no se lograron identificar diferencias significativas por género. Sin embargo, los hombres presentaron una correlación con los estilos reflexivo y pragmático y las mujeres encontraron una relación inversa entre los estilos activo y teórico. Por lo que dicha investigación, se concluye que los estudiantes prefieren la aplicación de ideas, pues éstas les permiten una mejor comprensión del tema, lo cual brinda una mejor perspectiva al docente al momento de la planeación educativa y le brinda un mejor desenvolvimiento académico, por lo que el docente o los mismos estudiantes deben de tenerlas en cuenta.

Otro estudio para conocer las diferencias de género en estrategias y estilos de aprendizaje es el realizado por Cano (2000), que consistía en aplicar a 991 estudiantes cuatro pruebas, L.A.S.S.I., (Learning and Study Strategies Inventory) el cual evalúa 10 escalas: Actitud, Ansiedad, Autocomprobación, Concentración, Administración del Tiempo, Estrategias de Examen, Ayudas de Estudio, Motivación, Procesamiento de la Información y Selección de Idas Principales; el I.L.P. Inventory of Learning Processes o Inventario de Procesos de Aprendizaje ; A.S.I. Approaches to Studying Inventory (Inventario de Enfoques de Estudio), y L.S.Q. Learning Styles Questionnarie o Cuestionario de Estilos de Aprendizaje. Además, realizaron el análisis MANOVA para las variables género, tipo de carrera y curso. El resultado mostró efectos estadísticamente significativos para esas variables como para la interacción génerocarrera, y dio como conclusión que sí existen diferencias entre las estrategias y los estilos de aprendizaje acorde al género y que éstas también dependen de la carrera seleccionada. Por ejemplo, en las carreras de ciencias, ciencias sociales y letras, las alumnas tienen miedo al fracaso; los alumnos, sin embargo, muestran más actitudes negativas hacia el aplicar 


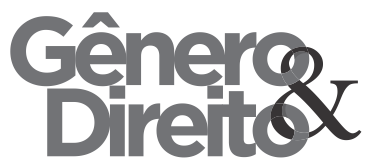

técnicas de estudio.

\section{Metodología}

La presente investigación es un estudio descriptivo, la muestra es no aleatoria dirigida (Hernández, Fernández, y Baptista, 2006) y compuesta por los alumnos de nuevo ingreso, a los que se les aplicó la prueba EDAOM (Inventario de Estilos de Aprendizaje y Orientación Motivacional), la que contestaron totalmente. La muestra se encontraba conformada por 97 estudiantes de la Licenciatura en Administración, pertenecientes a las cohortes generacionales 2010, 2011 y 2012, distribuidos en 41 hombres y 56 mujeres, porcentualmente 42 y 58 respectivamente. La edad estaba comprendida entre 17 y 28 años, del cual el $68 \%$ del total de la muestra contaba con una edad de 17 a 19 años.

INVENTARIO DE ESTILOS DE APRENDIZAJE Y ORIENTACIÓN MOTIVACIONAL (EDAOM).
Se utilizó el inventario de Estilos de Aprendizaje y Orientación Motivacional (EDAOM), en el cual los estudiantes realizan una autovaloración de sus estrategias de aprendizaje y orientaciones motivacionales que utilizan para sus estudios.

Está compuesto por 89 reactivos tipo Likert correspondientes a 4 escalas: a) Estilos de adquisición de la información,

b) Estilos de recuperación de la información, c) Estilos de procesamiento de la información y d) Estilos de autorregulación metacognitiva y metamotivacional, la figura 1 muestra la estructura del EDAOM (Castañeda, 2004).

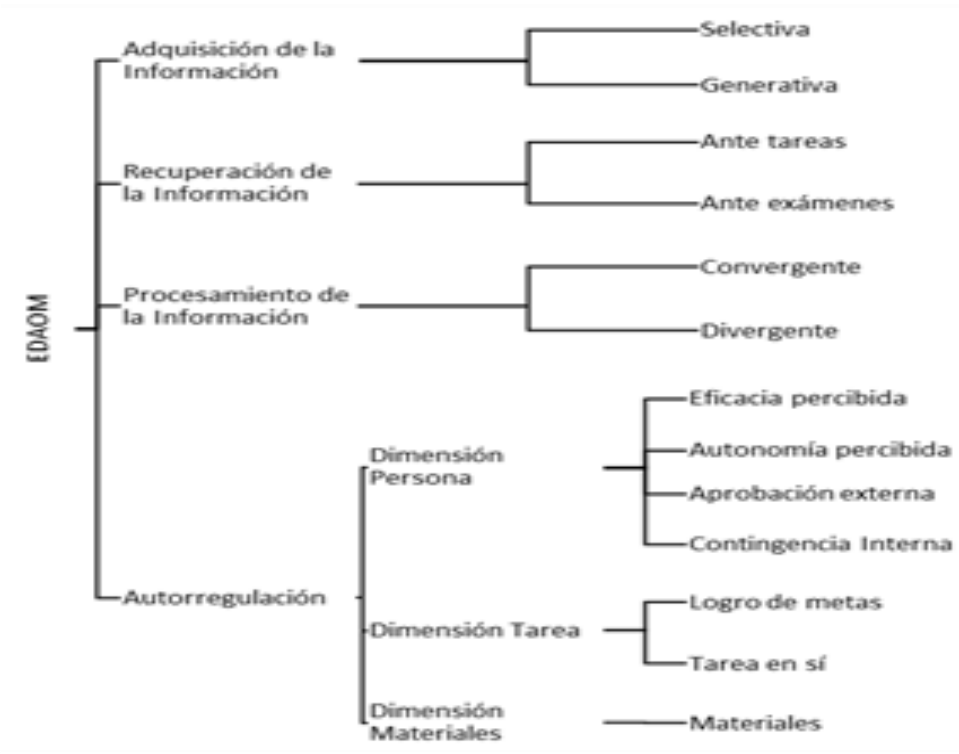

Figura 1 Estructura del EDAOM.

Fuente: Castañeda (2004). 
Las estrategias de Adquisición de la Información están compuestas por:

- Selectivas: Son estrategias de procesamiento superficial de lo que se está aprendiendo, consiste en seleccionar la información importante de la manera más rápida y completa, utilizando estrategias usadas como la hojeada y las lecturas rápidas que tratan de identificar lo más importante y subrayarlo, repasando el material difícil de comprender (Castañeda y López, 1999 citado en Niño, 2013).

- Generativas: Son estrategias de procesamiento profundo de la información adquirida, manejando algunas estrategias de aprendizaje como las analogías para relacionar conceptos, mapas mentales para análisis de información, cuadros sinópticos que estructuran la información, etc. (Castañeda y López, 1999 citado en Niño, 2013).

Las estrategias de Recuperación de la Información aprendida permiten preactivar, reactivar y mantener activada la información para poder ser utilizada posteriormente, algunas estrategias de aprendizaje empleadas son: la relectura, generar preguntas para evaluar lo comprendido, recordar una imagen, las cuales se realizan ante diferentes tareas académicas y durante los exámenes (Castañeda y López, 1999 citado en Niño, 2013).

Las estrategias de procesamiento de la información se relacionan con estructurar la información aprendida y razonar o criticar lo aprendido. Algunas de estas estrategias son: agrupamiento de conceptos en categorías, relacionar ideas entre sí, estructurar el conocimiento mediante mapas conceptuales (Castañeda y López, 1999 citado en Niño, 2013). En ellas, el procesamiento de la información se realiza de manera convergente por lo que la información aprendida se reproduce $\mathrm{y}$ en el procesamiento divergente se crean producciones innovadoras y se piensa críticamente sobre lo aprendido.

$$
\text { Las estrategias de }
$$
autorregulación metacognitiva y metamotivacional incluyen actividades que permite al estudiante planear, evaluar y regular si ha logrado cumplir las metas 
de aprendizaje (Castañeda y López, 1999 citado en Niño, 2013). En ella, las estrategias de tarea de aprendizaje se enfocan a la Orientación a la tarea en sí y Orientación al logro de metas. Y las estrategias de los Materiales de Aprendizaje se emplean en cuanto a su utilidad para proporcionar el aprendizaje eficiente.

Los criterios para la interpretación de los resultados de la evaluación del EDAOM se muestran en la Tabla 1:

\begin{tabular}{ll}
\hline Puntaje & Interpretación \\
\hline $100-76$ & Indica que el estudiante tiene un buen \\
& desarrollo de estrategias de aprendizaje \\
$75-56$ & Indica un resultado regular, por lo cual hay \\
& que reforzar las estrategias de aprendizaje \\
& correspondientes. \\
& Indica un resultado insuficiente, por lo cual \\
$55-0$ & hay que entrenar las estrategias de \\
& aprendizaje \\
\hline
\end{tabular}

Tabla 1 Criterios de interpretación de resultados de la evaluación del EDAOM. Fuente: Castañeda (2004)

\section{Estadísticos Descriptivos}

En la Tabla 2 se muestra los estadísticos descriptivos de las estrategias de aprendizaje de adquisición, recuperación y procesamiento de la información de la muestra. De acuerdo con los criterios de interpretación de los resultados del EDAOM se observa que, en general, los estudiantes tienen un buen desarrollo de las estrategias de aprendizaje Generativa,
Recuperación de la información durante los exámenes y de Procesamiento Convergente; y, en cuanto a las estrategias selectivas, Recuperación de la información ante las tareas y Procesamiento Divergente, se requiere reforzar estas estrategias de aprendizaje. Así mismo, se observa que no se obtuvo un resultado insuficiente en alguna de las estrategias de 
aprendizaje que indique que se requiere

asesoría y apoyo en ellas.

\begin{tabular}{|c|c|c|c|c|}
\hline $\begin{array}{l}\text { Estrategia de } \\
\text { Aprendizaje }\end{array}$ & Media & $\begin{array}{l}\text { Desviación } \\
\text { estándar }\end{array}$ & Mínimo & Máximo \\
\hline Selectiva & 69.86 & 10.167 & 46 & 94 \\
\hline Generativa & 78.48 & 11.311 & 49 & 100 \\
\hline $\begin{array}{l}\text { Recuperación } \\
\text { ante Tarea }\end{array}$ & 69.98 & 9.009 & 47 & 90 \\
\hline Recuperación & & & & \\
\hline $\begin{array}{l}\text { Durante } \\
\text { Exámenes }\end{array}$ & 80.59 & 10.414 & 60 & 100 \\
\hline Convergente & 76.41 & 11.374 & 49 & 97 \\
\hline Divergente & 74.85 & 11.407 & 46 & 100 \\
\hline
\end{tabular}

Tabla 2 Estadísticos descriptivos de estudiantes de Licenciatura en Administración

La Tabla 3 y Tabla 4 muestran los estadísticos descriptivos obtenidos acorde al género, que brindan un resultado en el que los estudiantes de género masculino obtienen un mejor resultado en el estilo de adquisición de la información, y obtienen mejores resultados en el uso de estrategias selectivas y generativas, manejando un puntaje de 70.68 y 79.29 a diferencia del género femenino. En lo referente al estilo de recuperación de la información aprendida ante las tareas, las estudiantes de género femenino obtienen un mejor desempeño con un 70.16 y los estudiantes de género masculino emplean más las estrategias de recuperación de la información durante los exámenes con un 82.20.

En cuanto al tipo de procesamiento de la información convergente, las estudiantes del género femenino obtuvieron un resultado mayor a 
Periódico do Núcleo de Estudos e Pesquisas sobre Gênero e Direito Centro de Ciências Jurídicas - Universidade Federal da Paraíba V. 7 - $\mathrm{N}^{\mathrm{o}} 04$ - Ano 2018 - Spanish Edition ISSN | 2179-7137 | http://periodicos.ufpb.br/ojs2/index.php/ged/index

los del género masculino, aunque esta diferencia es mínima al obtener 76.57 y 76.20 respectivamente; en cuanto a las estrategias de procesamiento de la información divergente, los estudiantes del género masculino obtuvieron un resultado mayor a las estudiantes del género femenino, aunque la diferencia es mínima al obtener 75.07 y 74.68 respectivamente.

\begin{tabular}{|c|c|c|c|c|}
\hline $\begin{array}{l}\text { Estrategia de } \\
\text { Aprendizaje }\end{array}$ & Media & $\begin{array}{l}\text { Desviación } \\
\text { estándar }\end{array}$ & Mínimo & Máximo \\
\hline Selectiva & 70.68 & 8.912 & 51 & 94 \\
\hline Generativa & 79.29 & 11.714 & 57 & 97 \\
\hline $\begin{array}{l}\text { Recuperación } \\
\text { ante Tarea }\end{array}$ & 69.73 & 8.823 & 47 & 87 \\
\hline Recuperación & & & & \\
\hline Durante & 82.20 & 11.059 & 60 & 100 \\
\hline Exámenes & & & & \\
\hline Convergente & 76.20 & 11.201 & 49 & 97 \\
\hline Divergente & 75.07 & 12.789 & 51 & 100 \\
\hline
\end{tabular}

Tabla 3 Estadísticos descriptivos de estudiantes de género Masculino de la Licenciatura en Administración

\begin{tabular}{lllll}
\hline $\begin{array}{l}\text { Estrategia de } \\
\text { Aprendizaje }\end{array}$ & Media & $\begin{array}{l}\text { Desviación } \\
\text { estándar }\end{array}$ & Mínimo & Máximo \\
\hline Selectiva & 69.25 & 11.036 & 46 & 94 \\
Generativa & 77.89 & 11.076 & 49 & 100 \\
$\begin{array}{l}\text { Recuperación } \\
\text { ante Tarea }\end{array}$ & 70.16 & 9.218 & 50 & 90 \\
and & & & &
\end{tabular}




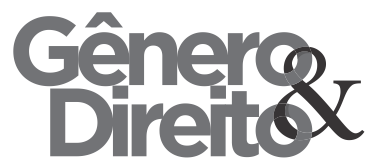

Periódico do Núcleo de Estudos e Pesquisas sobre Gênero e Direito Centro de Ciências Jurídicas - Universidade Federal da Paraíba V. 7 - $\mathrm{N}^{\mathrm{o}} 04$ - Ano 2018 - Spanish Edition ISSN | 2179-7137 | http://periodicos.ufpb.br/ojs2/index.php/ged/index

Recuperación

$\begin{array}{lcccc}\text { Durante } & 79.41 & 9.849 & 60 & 100 \\ \begin{array}{l}\text { Exámenes } \\ \text { Convergente }\end{array} & 76.57 & 11.598 & 54 & 97 \\ \text { Divergente } & 74.68 & 10.396 & 46 & 100\end{array}$

Tabla 4 Estadísticos descriptivos de estudiantes de género Femenino de la Licenciatura en Administración

\section{Análisis Correlacional}

Para detectar asociaciones entre las variables seleccionadas del EDAOM se realizó una correlación de Pearson. La figura 2 muestra las asociaciones entre las subescalas selectiva, generativa, recuperación ante tareas, recuperación ante exámenes, procesamiento convergente y procesamiento divergente. Los índices de correlación de Pearson muestran que las seis subescalas presentan asociaciones entre ellas.

- La subescala Selectiva está asociada fuertemente con la subescala Convergente

- La Generativa correlaciona fuertemente con Divergente, y la subescala de Recuperación Ante Exámenes con Generativa y Recuperación ante Tareas con Generativa.

- La subescala Convergente está correlacionada fuertemente con la subescala Divergente.

- La subescala Recuperación ante Exámenes correlaciona fuertemente con Convergente

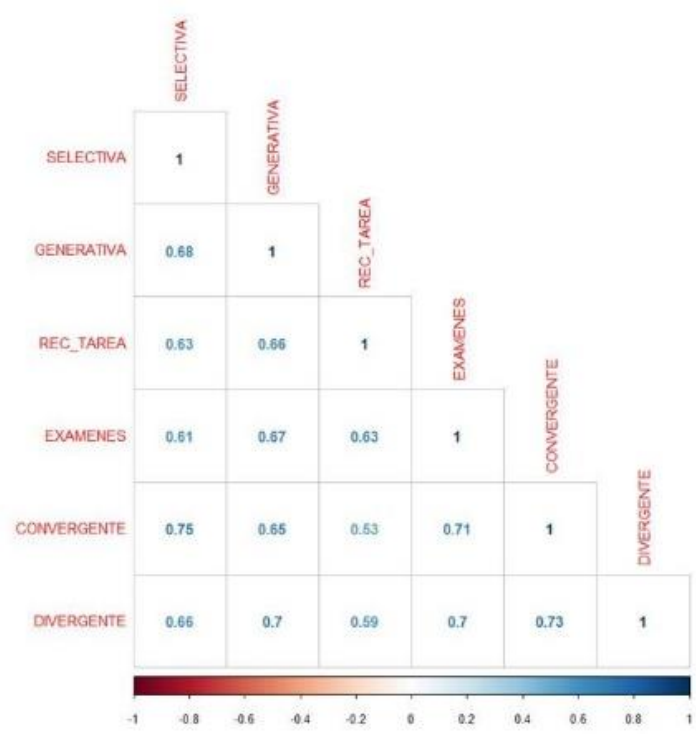


Figura 2. Asociaciones entre las escalas de adquisición, recuperación y procesamiento de la información

De acuerdo a las correlaciones obtenidas se concluye que estas estrategias de aprendizaje no son excluyentes, es decir que los estudiantes tienen desarrollada las diferentes estrategias de aprendizaje y esto es bueno debido a que la implementación de este serio acorde a la necesidad que se requiera en un determinado momento de su aprendizaje.

\section{Representación De Radar De La Muestra}

Las representaciones gráficas de los datos son de gran valor a la hora de extraer conocimiento útil, sus ventajas son la visualización de la información de una forma sencilla, rápida y directa (Sáez, Soria y Martín, 2015).

La representación radial se ha convertido en una alternativa efectiva en el soporte de visualizaciones de alta dimensionalidad y con grandes volúmenes de datos, con mayor capacidad de escalabilidad y con un mejor aprovechamiento del espacio disponible (Alvarado, 2010).

Para representar los resultados obtenidos de la aplicación del EDAOM a los estudiantes de la Licenciatura en Administración, se realizó una gráfica radial en la cual cada uno de los ejes representa una subescala: las coordenadas de las posiciones alcanzadas de la media de cada subescala por género que componen la muestra y el mínimo ideal de un buen resultado del EDAOM. En la figura 3 se puede observar que: 


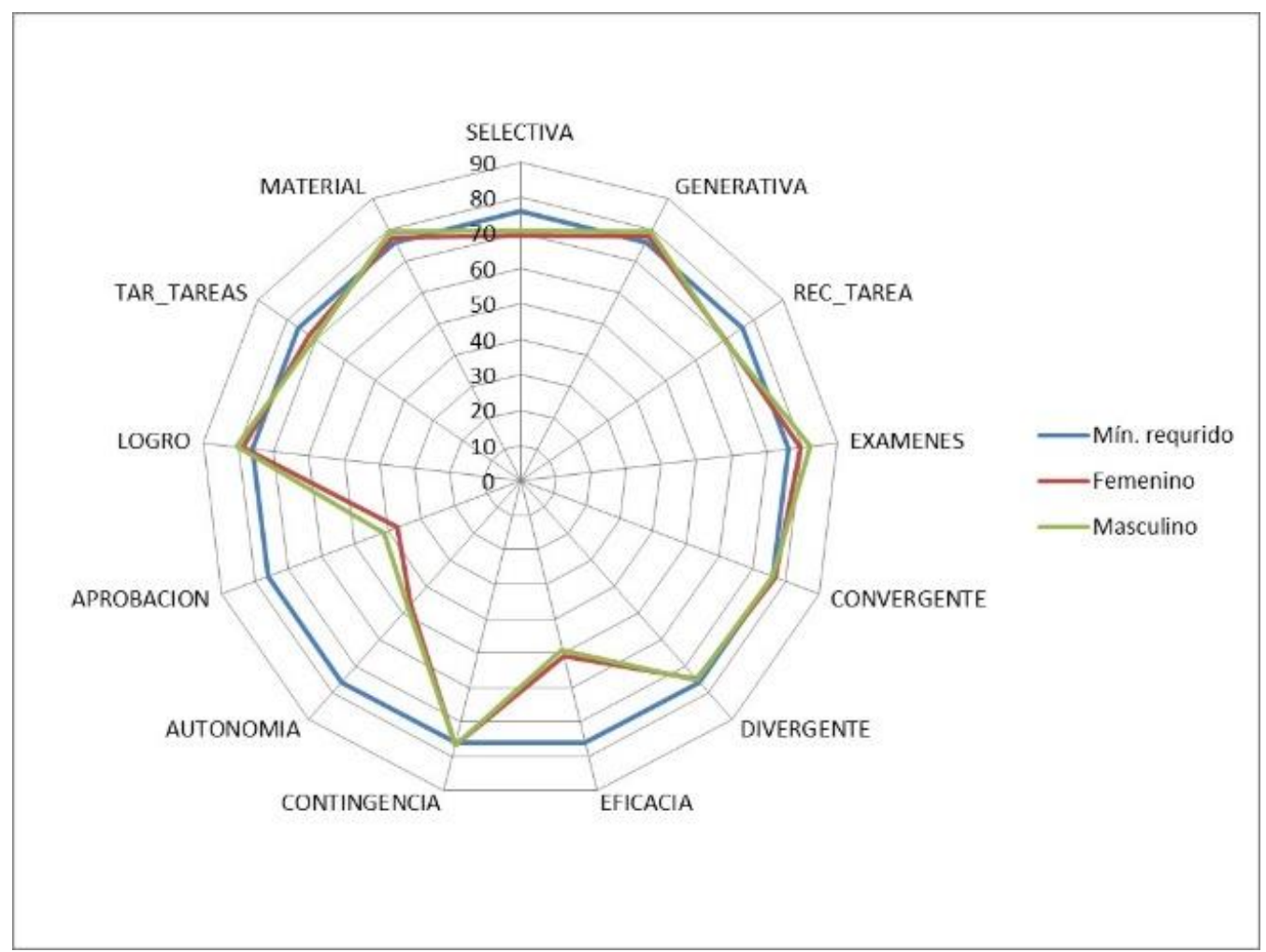

Figura 3 Representación radial de las subescalas del EDAOM de estudiantes de la Licenciatura en Administración

Las subescalas Generativa, contingencia percibida los hombres Recuperación durante Exámenes, obtienen un puntaje bueno, mientras que Orientación al Logro y Materiales se las mujeres obtienen un resultado encuentran en un buen nivel que no levemente inferior al de los hombres. requieren sean reforzadas.

Así mismo en la subescala

Las subescalas Convergente $\mathrm{y}$ Selectiva, Orientación a la Tarea, Divergente los hombres se encuentran en Recuperación ante diversas tareas ambos un buen nivel y las mujeres obtienen un resultado regular, aunque están muy cercas de la frontera del puntaje mínimo para obtener un buen resultado. géneros obtuvieron un resultado regular indicando que éstas requieren ser reforzadas.

En la escala de Orientación a la En cuanto a la subescala Aprobación Externa los estudiantes del 
género masculino obtuvieron un mejor resultado que las mujeres, sin embargo, ambos géneros están por debajo del puntaje mínimo requerido, por lo cual, también requiere reforzamiento.

En la subescala Eficacia Percibida, las mujeres obtuvieron un mejor resultado que los hombres, aunque ambos requieren que se refuerce esta orientación meta motivacional.

En cuanto a la Autonomía percibida, el resultado obtenido es muy similar para ambos géneros; aunque la diferencia en el resultado es mínima, los hombres tienen un mejor resultado.

En base a los resultados visualizados se concluye que los estudiantes evaluados, presentan un estilo de adquisición de la información generativa donde el estudiante puede usar imágenes de lo que está aprendiendo para hacerlo más comprensible, por lo cual, el procesamiento que usan es divergente, $\mathrm{y}$, en el cual, son capaces de crear y pensar críticamente sobre lo que aprenden; y, en consecuencia, obtienen un buen resultado en la recuperación de la información durante los exámenes.

\section{Representación Radial De Casos}

Para mostrar otra representación de la gráfica radial con datos multivariados, se presenta la Figura 4 de cuatro casos, de estudiantes elegidos al azar del total de la muestra, de los cuales dos son de género femenino y dos del género masculino, mostrando a continuación, la descripción de la gráfica:

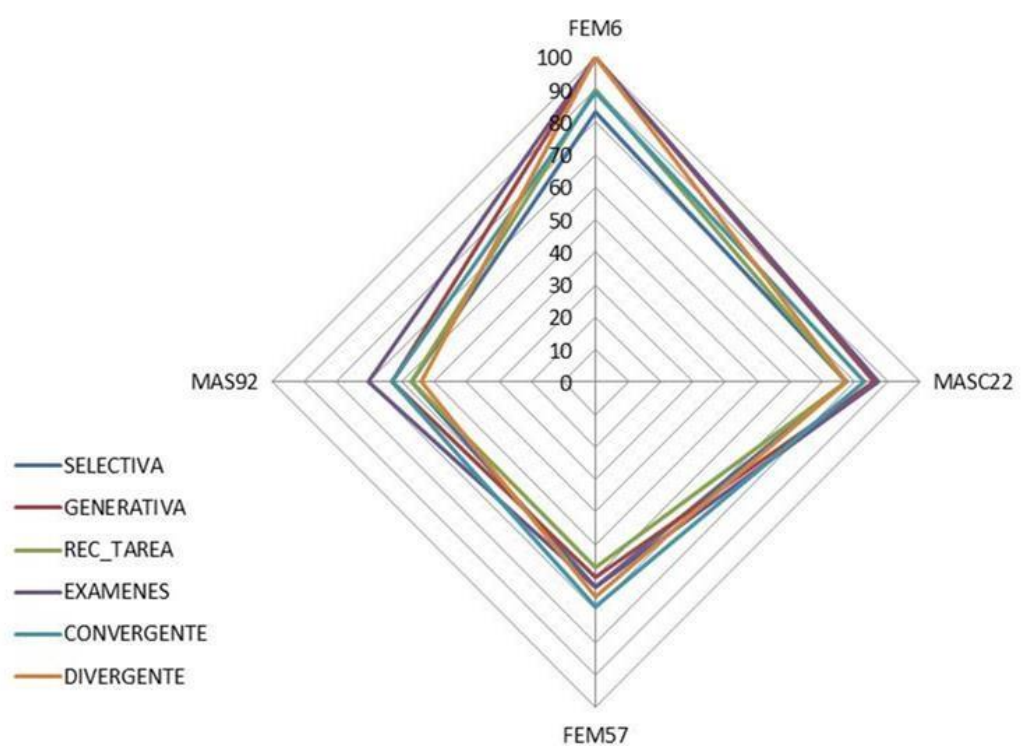




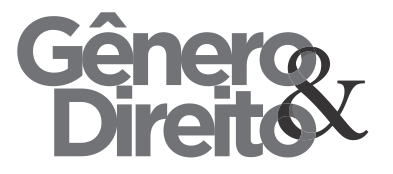

Periódico do Núcleo de Estudos e Pesquisas sobre Gênero e Direito

Centro de Ciências Jurídicas - Universidade Federal da Paraíba

V. 7 - No 04 - Ano 2018 - Spanish Edition

ISSN | 2179-7137 | http://periodicos.ufpb.br/ojs2/index.php/ged/index

Figura 4 Representación de datos multivalentes de estudiantes de Lic. en Administración

En la figura 3 se observa que el caso FEM6 obtuvo los mejores resultados en las estrategias generativa y divergente, y la más baja es selectiva. El caso MAS22 obtiene los puntajes más altos en generativa y recuperación de la información durante exámenes y los más bajos en selectiva, recuperación de la información ante diversas tareas y divergente. El caso FEM57 muestra que el puntaje más alto es convergente y el más bajo la recuperación de la información ante tareas. Para el caso FEM92 se observa que el resultado más alto lo obtuvo en recuperación durante exámenes y el más bajo es divergente.

De manera general se observa que el caso del estudiante de género femenino FEM6 es quien obtiene los mejores puntajes, y que el caso del estudiante de género masculino MAS92 es quien ha tiene los resultados más bajo de los cuatro.

El caso FEM6 muestra que en todas las subescalas ha obtenido un buen resultado, que la adquisición de información predominante es la generativa, en la cual emplea estrategias para realizar representaciones graficas de lo que aprende para comprenderlo, el procesamiento de la información es divergente al crear y pensar críticamente sobre lo aprendido, facilitándose la recuperación ante exámenes.

El caso MAS22 ha obtenido buenos resultados, se observa que para la adquisición de conocimiento utiliza las estrategias generativas, que la recuperación de la información durante exámenes es buena como consecuencia de usar guías, imágenes que le permitan recordar y recuperar fácilmente lo aprendido, y que utiliza más estrategias de procesamiento convergente, hablando en este caso de la técnica de memorización sobre lo que estudia, aun cuando también obtuvo un resultado bueno en el procesamiento convergente.

\section{Los casos FEM57 y MAS92} obtuvieron resultados regulares por lo cual requieren que se refuercen las estrategias de aprendizaje de: adquisición, recuperación y procesamiento de la información. 


\section{Desempeño Por Género}

En este estudio se considera la variable desempeño académico por género, debido a que cada estudiante tiene diferente personalidad, aprende y usa sus propias estrategias de aprendizaje, obteniendo resultados diferentes en cuanto a su desempeño académico (Acevedo y Rocha, 2011). El desempeño general que presenta esta muestra se presenta en la Tabla 4, en la cual se observa que un $36 \%$ de estudiantes obtuvo un desempeño bajo, un poco más de la mitad el $51 \%$ obtuvo un desempeño bueno y un $13 \%$ obtuvo un desempeño regular. En cuanto al desempeño obtenido por género se observa que, las mujeres obtuvieron un mejor desempeño que los hombres al obtener un $33 \%$ de desempeño bueno frente a un $18 \%$ de los hombres, y el $21 \%$ de los hombres obtuvieron un desempeño bajo frente a un $15 \%$ de las mujeres, tan solo un $9 \%$ de mujeres obtuvieron un resultado regular frente a un $4 \%$ de los hombres.

En esta muestra el género femenino obtuvo un mejor rendimiento o desempeño académico.

\begin{tabular}{lllc}
\hline \multirow{2}{*}{ Desempeño } & Género & & $\%$ \\
& F & M & Total \\
\hline Bajo & $15 \%$ & $21 \%$ & $36 \%$ \\
Regular & $9 \%$ & $4 \%$ & $13 \%$ \\
Bueno & $33 \%$ & $18 \%$ & $51 \%$ \\
Total & $58 \%$ & $42 \%$ & $100 \%$ \\
\hline
\end{tabular}

Tabla 4 Resultados de desempeño por género

\section{Conclusiones}

Los resultados obtenidos en esta investigación muestran que las diferencias del uso de las estrategias de aprendizaje entre hombres y mujeres, se da mayormente en las estrategias de recuperación de la información, en la cual los hombres manejan mayor destreza durante los exámenes, significando que se preparan para presentar los exámenes. Con este resultado se infiere que los hombres están orientados a la búsqueda de logros 
académicos, reflejándose en el efecto obtenido en la orientación meta motivacional donde los hombres obtienen un mejor resultado al logro de metas académicas o acreditación de una asignatura.

En cambio, las mujeres obtienen un mejor resultado en la orientación a la tarea en sí y en materiales, haciendo un uso adecuado y eficiente del mismo y por consiguiente obtienen un mejor resultado en la evaluación de la eficacia de las estrategias empleadas para lograr un buen resultado en las tareas de aprendizaje. Cabe aclarar que las diferencias en los resultados de las estrategias usadas entre hombres y mujeres son mínimas. Sin embargo, la mayor diferencia se da en los resultados del desempeño académico la cual se refleja mejor en las mujeres.

Este tipo de estudios se presenta importante, ya que sirve como apoyo a los Programas de Tutorías establecidos en las Instituciones de Educación Superior, que tienen el objetivo de servir de apoyo para una toma de decisiones acertadas y pertinentes y brinden la asesoría e intervención justa y necesaria para que el estudiante se desenvuelva académicamente de manera apropiada.

\section{Referencias Bibliográficas}

Acevedo, D., Cavadia, S. y Alvis, A., (2015). Estilos de Aprendizaje de los Estudiantes de la Facultad de Ingeniería de la Universidad de Cartagena (Colombia). Form. Univ. [online], vol.8, n.4, pp.15-22. ISSN 0718-5006. http://dx.doi.org/10.4067/S071850062015000400003.

Acevedo, C., Rocha, F. (2011). Estilos de aprendizaje, género y rendimiento académico. Revista de Estilos de Aprendizaje. Journal of Learning Styles, vol. 4, no 8, pp.71-84. Recuperado de: http://learningstyles.uvu.edu/index.php/jls

\section{/article/view/65}

Alvarado, J.C. (2010). Visualización multidimensional, nuevas técnicas de exploración de datos y representación de información, Revista Memorias, vol. 8, núm. 14, pp. 72-84. Recuperado de: http://revistas.ucc.edu.co/index.php/me/ar $\underline{\text { ticle/view/168/169 }}$ 
Beltrán, J. A. (2003). Estrategias de aprendizaje. Revista de Educación.

Doná, S. M., Lopetegui, M. S., Rossi Casé, Madrid, (332), 55-73. Recuperado de: http://201.147.150.252/handle/123456789 $\underline{1391}$

Cano, F., (2000). Diferencias de género en estrategias y estilos de aprendizaje. Psicothema, 360-367. Recuperado de: http://www.redalyc.org/articulo.oa?id=72 L. E., Neer, R. H., (2010). Estrategias de aprendizaje y rendimiento académico según el género en estudiantes universitarios (En línea). Revista de Psicología (La Plata), (11): 199-211. Recuperado en: http://www.memoria.fahce.unlp.edu.ar/art 712306.

Castañeda, S., (2004). Educación, aprendizaje y cognición. Teoría en la práctica. El Manual _revistas/pr.4846/pr.4846.pdf

Hernández, R.; Fernández-Collado, C.; Baptista, P., (2010). Metodología de la investigación. México, D.F., (5a ed.) México: McGraw-Hill Interamericana.

Moderno S.A. de C.V. pp 277 - 298. ISBN: 9789707290884

Costa, S., Tabernero, C., (2012). Rendimiento académico y autoconcepto en estudiantes de educación secundaria obligatoria según el género. Revista Iberoamericana de Psicología y Salud, vol. 3, núm. 2, julio, 2012, pp. 175-193. Sociedad Universitaria de Investigación en Psicología y Salud A Coruña, España Recuperado de: http://www.redalyc.org/pdf/2451/245124 456006.pdf

Niño, M. (2013). La Relación Estilos de Aprendizaje y Rendimiento Académico en Alumnos de una Facultad de la UANL. Tesis de doctorado. Universidad Autónoma de Tamaulipas. Recuperada de: http://bibliotecadigital.tamaulipas.gob.mx /archivos/descargas/af52794ae030d3d567 e2858991a8389760a1fc22.pdf

Sáez, M.A., Soria, E., Martín, J. D., (2015). Diseño e implementación de una aplicación en Processing para la representación visual de datos 
Periódico do Núcleo de Estudos e Pesquisas sobre Gênero e Direito Centro de Ciências Jurídicas - Universidade Federal da Paraíba V. 7 - $\mathrm{N}^{\mathrm{o}} 04$ - Ano 2018 - Spanish Edition ISSN | 2179-7137 | http://periodicos.ufpb.br/ojs2/index.php/ged/index

multidimensionales utilizando técnicas de

Minería de Datos. Universidad de

Valencia. Recuperado de:

http://roderic.uv.es/handle/10550/49206?s

$\underline{\text { how }=\text { full }}$

Vélez Van Meerbeke, A., Roa González,

C. N., (2005). Factores asociados al rendimiento académico en estudiantes de medicina. Educación médica, 8(2), 24-32.

Recuperado

de:

http://scielo.isciii.es/scielo.php?pid=S157

5 -

18132005000200005\&script=sci_arttext

\&tlng=pt 